\title{
INTERVENCIÓN ESPECIALIZADA EN MALTRATO INFANTIL EN CHILE EN CONTEXTO DE CRISIS POR COVID-19: SIGNIFICADOS DE LOS PROFESIONALES INTERVINIENTES
}

\author{
SPECIALIZED INTERVENTION IN CHILD ABUSE AND MALTREATMENT IN \\ CHILE IN THE CONTEXT OF CRISIS DUE TO COVID-19: MEANINGS OF THE \\ PROFESSIONALS WHO PARTICIPATE IN THE INTERVENTION
}

\author{
Cristián Pinto-Cortez \\ Universidad de Tarapacá \\ Arica - Chile \\ Valeria Arrendondo Ossandón \\ Corporación ONG Paicabi \\ Viña del Mar-Chile \\ Gonzalo Lira \\ Universidad de Valparaíso \\ Valparaíso-Chile
Paula Lobos Sucarrat
Corporación ONG Paicabi \\ Viña del Mar-Chile \\ Carolina Saavedra \\ Corporación ONG Paicabi \\ Viña del Mar-Chile \\ Cristóbal Guerra \\ Universidad Santo Tomás \\ Viña del Mar-Chile \\ Recibido noviembre de 2020/Received November, 2020 \\ Aceptado abril de 2021/Accepted April, 2021
}

\begin{abstract}
RESUMEN
La crisis sociosanitaria por COVID-19 ha afectado la forma en que los centros especializados en apoyo a víctimas de maltrato infantil realizan su labor. El formato de atención ha pasado desde lo presencial a lo online, con los desafíos que eso conlleva tanto en el ámbito práctico como en el ámbito técnico-profesional. Las familias más vulnerables presentan mayores problemas de conexión -con el consecuente aislamiento- y mayores estresores que pueden empeorar la relación al interior de la familia, aumentando la complejidad de las intervenciones. En este contexto el objetivo del estudio fue describir los significados de los profesionales de centros especializados en la atención a víctimas de maltrato infantil respecto de la intervención especializada desarrollada durante la crisis por COVID-19. Participaron 42 profesionales de estos centros, quienes respondieron una entrevista
\end{abstract}


semiestructurada online. Los resultados del análisis de contenido cualitativo dan cuenta de significados asociados a los riesgos asociados a la intervención en este contexto (para los niños y sus familias, para la intervención, asociados a la precarización de condiciones sociomateriales, baja conectividad y confinamiento). No obstante, los participantes también destacan oportunidades y recursos en la intervención (la capacidad de adaptar las intervenciones al nuevo contexto y el trabajo de equipo). Todo lo anterior resulta de utilidad en lo inmediato de forma de visibilizar riesgos y oportunidades en una crisis que parece ser que será más larga de lo originalmente pensado. Es necesario actuar rápidamente para realizar las modificaciones pertinentes en las intervenciones, de forma de disminuir los riesgos y potenciar las oportunidades en beneficio de los niños, niñas y adolescentes.

Palabras Clave: COVID-19, Intervención psicosocial, Maltrato infantil, Abuso sexual.

\section{ABSTRACT}

The pandemic by COVID-19 affected the workplace of psychosocial teams for the support of child abuse victims. The ways of attention have passed from the face to face to the online sessions, with the challenges that this entails in both the practical and technical, protective. The most vulnerable families present greater problems of internet connection -with the consequent isolation-and greater stress that can worsen the relationship within the family, increasing the complexity of the interventions. In this context, the aim of the study was to describe the meanings of the professionals in centers specialized in the care of child abuse victims with respect to the specialized intervention developed during the crisis by COVID-19. Forty-two professionals from these centers participated and answered a semi-structured online interview. The results of the analysis of qualitative content give an account of the meanings associated to the risks associated to the intervention in this context (for the children and their families, for the intervention, associated to the precarization of sociomaterial conditions, low connectivity and confinement). However, participants also highlight opportunities and resources in the intervention (the capacity to adapt the interventions to the new context and teamwork). All of the above is useful in the immediate term in order to make risks and opportunities visible in a crisis that seems to be longer than originally thought. It is necessary to act quickly in order to make the pertinent modifications in the interventions so as to reduce the risks and enhance the opportunities for the benefit of children and adolescents

Keywords: COVID-19, Psychosocial intervention, Child abuse, Sexual abuse.

\section{INTRODUCCIÓN}

La pandemia mundial por la enfermedad del coronavirus COVID-19 (19 Coronavirus Disease) es un fenómeno global sin precedentes (Liang et al., 2020). Esta enfermedad ha afectado a todos los países y a todos los grupos que componen las sociedades, sin distinción de sexo, edad, etnia u orientación sexual (Novel, 2020). A pesar de ser una enfermedad sin cura conocida, los equipos de salud luchan a diario por ayudar a superar las consecuencias de esta pandemia en quienes la padecen de forma leve, grave o crítica. Además de las secuelas físicas que produce el COVID-19, se ha constatado que la enfermedad del coronavirus produce un impacto social negativo en la población general. Por ejemplo, investigaciones acerca de los efectos psicológicos han demostrado que los pacientes diagnosticados con el COVID-19 presentan síntomas como arrepentimiento ante la infección (¿por qué no hice lo que tenía que hacer?), resentimiento (¿por qué me pasó esto a mí?), soledad e impotencia, depresión, ansiedad, fobia, irritabilidad y falta de sueño. Algunos pacientes pueden presentar crisis de pánico (Liang et al., 2020). Las evaluaciones psicológicas que se han realizado en las salas de aislamiento (China) demostraron que alrededor del $48 \%$ de los pacientes con COVID-19 manifestaron estrés psicológico durante los primeros ingresos a los sistemas sanitarios, principalmente una respuesta emocional al estrés. Asimismo, el porcentaje de delirio es alto entre los pacientes críticamente enfermos. Incluso se ha informado de un tipo de encefalitis inducida por el SARS-CoV-2 que provoca síntomas psicológicos como la inconsciencia y la irritabilidad (Liang et al., 2020). Sin duda estos síntomas requieren una intervención psicológica permanente en las salas de hospitalización. Sin embargo, los efectos psicológicos no se presentan exclusivamente en las personas que sufren la enfermedad, sino también en quienes padecen las estrictas medidas sanitarias que implementan los Estados para la erradicación de la enfermedad, como por ejemplo las medidas de confinamiento, la paralización total de actividades, la cuarentena obligatoria o la cuarentena voluntaria. Investigaciones realizadas en Australia, China, Canadá, Estados Unidos y Corea del Sur, han demostrado que la cuarentena ocasiona, en la población general, síntomas como frustración, aburrimiento, miedo a la infección, depresión, estrés e insomnio (Brooks et al., 2020). En ese contexto, el estrés se manifiesta en estados emocionales asociados al miedo, la ansiedad, la irritabilidad y la tristeza, pensamientos catastróficos, incertidumbre por la situación financiera y el estigma de portar la enfermedad (Brooks et al., 
2020). Aunque de manera diferente, estas reacciones afectan a niños, niñas, adolescentes y adultos. En esa línea, se reconoce el efecto de la pandemia en los niños en diferentes niveles: primero, tal como el resto de la población, los niños pueden reaccionar con síntomas de ansiedad y depresión debido a la crisis, a la pandemia y sus costos asociados (Dalton, Rapa y Stein, 2020; Danese et al., 2019). Estos autores destacan que, tal como los adultos, los niños comúnmente desarrollan síntomas ansiosos y depresivos como respuesta al aislamiento social de su grupo de pares y de actividades necesarias para su desarrollo integral, como el deporte y la educación; también presentan miedo al contagio o a las consecuencias que el COVID-19 puede tener para sus seres queridos (ej. Abuelos). En segundo lugar, diversos organismos internacionales han puesto en relieve el aumento del riesgo de maltrato infantil, incluso en hogares donde antes de la pandemia no existía (APA, 2020; United Nations, 2020). Así como lo demuestra una revisión sistemática tendiente a delimitar los efectos de cuarentenas en el contexto de COVID-19 (Brooks et al., 2020), los padres se encuentran expuestos a estresores adicionales que pueden afectar sus niveles de control de impulsos e irritabilidad. Existe abundante evidencia que esto, a su vez, se relaciona con el riesgo de que los padres tiendan a reaccionar de forma más violenta hacia sus hijos (Crouch y Behl, 2001; Kuntz, 2008). Esto es especialmente preocupante en los casos de niños que, ya desde antes del confinamiento provocado por el COVID-19, sufrían maltrato y abuso intrafamiliar (United Nations, 2020). En estos casos, el confinamiento preventivo impide a los niños tener acceso a redes de apoyo externas a la familia quienes podrían detectar y atender a los niños que sufren este tipo de violencia interpersonal (APA, 2020). En esa línea, el aislamiento social ha sido reportado como un importante factor de riesgo de violencia contra los niños en estudios previos (Gracia y Musitu, 2003; Tucker y Rodríguez, 2014). Por último, distintas organizaciones internacionales han destacado el mayor riesgo de abuso sexual online en tiempos de confinamiento y pandemia, debido al mayor tiempo que los niños pasarían en internet (Dorset Police UK, 2020; UNICEF, 2020).

Sin embargo, en el contexto internacional existen pocos estudios que indaguen sobre Niños, Niñas y Adolescentes (NNA) en condiciones proteccionales específicas vinculadas a la vulneración de sus derechos como maltrato infantil o abuso sexual en contextos de pandemias, esta limitación al acceso del conocimiento respecto del problema, pone de manifiesto la importancia de desarrollar investigación en esta área. En particular, a pesar de las cifras de informes oficiales y de las opiniones de expertos en el tema, en Chile no existen estudios que reflejen la realidad de los NNA insertos en el sistema de protección a la infancia, y tampoco de las experiencias de los profesionales que trabajan en esta área en contexto de pandemia. Un ejemplo de esta situación son los alrededor de 4.936 NNA que se encuentran bajo la protección del Estado de Chile por medio de un cuidado alternativo residencial, y que por disposición de la autoridad sanitaria, han permanecido desde inicios de esta crisis en confinamiento, observándose que a causa de la prolongada cuarentena y en atención de los factores sociales y de salud mental, se han debido elaborar paulatinamente planes para flexibilizar esta restricción (SENAME, 2020). Desde 1990, Chile se encuentra adscrito a la Convención internacional de los derechos de los NNA (ONU, 1989), por lo que el Estado tiene el deber de monitorear y promover el respeto de sus derechos, y prevenir o reparar cuando esos derechos son vulnerados. Creemos que el COVID-19 es un escenario que pone en jaque al sistema proteccional chileno y que, por tanto, debe ser estudiado.

Considerando lo anteriormente expuesto es que la presente investigación tuvo como objetivo describir los significados que construyen los profesionales que se desempeñan en centros especializados en la atención de NNA y sus familias, que han sufrido maltrato infantil y abuso sexual respecto de la intervención especializada desarrollada en el contexto de crisis sociosanitaria por COVID-19. Se entenderá por significados, los sentidos subjetivos producidos en la vida cultural humana, que se define ontológicamente como diferente de aquellos elementos sociales, biológicos, ecológicos y de cualquier otro tipo, relacionados entre sí en el complejo proceso de su desarrollo (González Rey, 2000).

\section{ORIENTACIÓN TEÓRICO-METODOLÓGICA}

El presente estudio correspondió a la orientación teórico metodológica de tipo cualitativo (Bernal, 2006) basado en los supuestos que sostienen el lugar central de los significados generados por los sujetos en el ejercicio de su producción discursiva (Earl, 
2000). Destacando, como señala Bernal (2006), el propósito de profundización de una temática, la descripción de sus particularidades, siempre en relación con el contexto de donde emergen, sin la búsqueda de la generalización de los resultados.

\section{Lugar de estudio}

La investigación se desarrolló en el marco de asociatividad que existe entre la Corporación Paicabi, la Universidad de Tarapacá y la Universidad de Valparaíso, en la promoción del compromiso responsable y la participación activa en la defensa de los derechos humanos de NNA. En este contexto, la Corporación Paicabi organizó el desarrollo de dos jornadas de trabajo con los profesionales de los Centros de Intervención Especializada en Maltrato Infantil pertenecientes a los 21 programas que desarrolla esta Corporación en la IV Región de Coquimbo y la V Región de Valparaíso en Chile. Las jornadas de trabajo se desarrollaron a base de la temática del impacto socioemocional de los NNA víctimas de malos tratos en el contexto COVID-19, vía plataforma de internet. En el espacio de estas jornadas se debatieron los tópicos de la intervención actual desarrollada por los profesionales intervinientes, la incorporación de la intervención remota, sus obstáculos y desafíos. Todos los participantes abordaron estas temáticas contestando un cuestionario que se les envió por correo electrónico previo a las jornadas y que lo remitieron por la misma vía al equipo ejecutor del estudio basados en los tópicos a indagar.

\section{Participantes}

Se trabajó con un muestreo por oportunidad (Hernández, Fernández y Baptista, 2014), ya que se incluyó en el estudio a los profesionales que participaron de las jornadas de trabajo organizadas por la Corporación Paicabi, en torno al tema del impacto socioemocional de los niños víctimas de malos tratos en contexto de COVID-19, desarrollada el 29 de abril y el 6 de mayo 2020, mediante plataforma internet a quienes se les invitó a este estudio. Los criterios de inclusión fueron: haber participado en estas jornadas internas de la Corporación Paicabi, ser parte del equipo estable de intervención de cada programa, y aceptar voluntariamente ser parte de la investigación firmando el consentimiento para dicho efecto.

Los participantes fueron 42 personas, mayoritariamente (de género femenino $81,6 \%$ vs. $18,4 \%$ de género masculino) de edades comprendidas entre 27 y 48 años $(M=34,47 ; D T=5,17)$. Se trató de profesionales (43,2\% trabajadores sociales y $56,8 \%$ psicólogos) de centros especializados de atención a niños que han sufrido maltrato infantil en sus distintas formas (negligencia, maltrato psicológico, físico, abuso sexual, testigo de violencia o combinaciones de los anteriores constituyendo perfiles de polivictimización) en la Zona Central de Chile. La experiencia profesional de los participantes varió entre 1 y 20 años $(M=7,63 ; D T=4,72)$. Todos los participantes habían continuado con su formación profesional después del pregrado: el 28,9\% había asistido a cursos o jornadas breves; el 63,1\% además había cursado diplomados o postítulos de mayor alcance; mientras que el 7,9\% había obtenido el grado de Magíster.

Técnicas de producción de información

Se utilizó como técnica de producción de información la entrevista semiestructurada, que tiene como propósito conseguir los significados que los informantes atribuyen a los temas en cuestión y obtener información lo más precisa posible en relación con un tema determinado (Díaz-Bravo, Torruco-García, Martínez-Hernández y Varela-Ruiz, 2013). En consideración, de las actuales condiciones de trabajo remoto de los profesionales en contexto COVID-19, se utiliza el medio escrito para la realización de las entrevistas. Todos los tópicos que se abordaron en esta entrevista fueron asociados al contexto de la crisis sociosanitaria COVID-19 y fueron: desafíos de la intervención, recursos personales y del equipo para la intervención, y obstáculos para la intervención en contexto de crisis.

\section{Procedimiento de análisis}

Se utilizó como técnica de análisis de datos el Análisis de Contenido Cualitativo (Fernández, 2002), utilizando el software para análisis cualitativo Dedoose 8.3.35, en el que se codificó el material escrito. El proceso de fragmentación fue el siguiente: 1. Determinación unidades de análisis para la reducción y simplificación de los datos. Alude a los fragmentos del texto que resultan relevantes de acuerdo con los objetivos del estudio y que implican la primera selección del material de análisis. 2 . Determinación de unidades de contexto, que son las bases de sentido localizables dentro del texto y que proveen el marco para interpretar las unidades de análisis. 3. Construcción de categorías que dan 
cuenta de agrupaciones de sentido a base de la integración entre la síntesis analítica del texto y las perspectivas teóricas. La categorización utilizó los criterios de exhaustividad, pertinencia, homogeneidad y exclusión mutua. Se trabajó con categorías a posteriori (Bardin, 2002). 4. Se desarrolló la validación de categorías o recategorización, que implicó que una vez creado el árbol categorial preliminar, se revisaron las relaciones y coherencias temáticas de cada categoría y subcategoría con el objetivo de evitar la reiteración y lograr los criterios de exhaustividad y exclusión mutua, reorganizando el árbol categorial otorgando mayor validez a esta construcción (Arbeláez y Onrubia, 2014).

\section{Consideraciones éticas}

El proyecto fue aprobado por el comité de ética de la ONG que patrocinó el estudio. Los participantes fueron convocados por medio de su correo electrónico institucional, aceptando voluntariamente participar del proceso, siendo requisito para ello su consentimiento escrito. La fase de recogida de datos se desarrolló entre el 29 de abril y el 5 de mayo del 2020. Las encuestas se enumeraron ( $\sin$ incluir datos de identificación de los participantes) y se ingresaron al programa de análisis de datos solo con identificación numérica resguardando su confidencialidad.

\section{Criterios de calidad}

El estudio se desarrolló teniendo a la vista los lineamientos para la calidad en investigaciones cualitativas conforme con los planteamientos de Blesa, Cabo De Guzmán, García, Gehring, Muñoz, Palacios y Rhodes (2014) acerca de conciencia de complejidad, perspectiva holística, autenticidad/credibilidad y reflexividad. Se enfatizó especialmente esta última, compartiendo la perspectiva de De la Cuesta (2015) en cuanto a que la reflexividad constituye el principal instrumento para lograr la calidad. En este sentido, la investigación contiene el conjunto de reflexiones colaborativamente construidas, donde se ha intentado el comprender la perspectiva de los investigadores participantes hasta llegar a un punto común que logra tener sentido el colectivo, desde las diferentes ideas y perspectivas críticas, con el común denominador básico en la consideración de NNA como sujetos de derechos y al respeto intrínseco por la persona humana, que condicionan el punto de vista de los investigadores involucrados y la perspectiva ética del trabajo involucrado en víctimas de violencia.

\section{RESULTADOS}

Los resultados dan cuenta de la producción de 486 unidades de análisis preliminares, las que fueron reducidas a 266 conforme con criterios de parsimonia y pertinencia. Posteriormente se realizó una primera categorización en que se definieron 13 categorías iniciales siguiendo criterios de redundancia, comunalidad, centralidad y ajuste temático, las que finalmente concluyeron 8 categorías y 24 subcategorías, en la fase de recategorización. La síntesis del proceso de reducción categorial realizada se presenta en la Figura 1:

\section{Figura 1}

Categorías y subcategorías de análisis. Elaboración propia

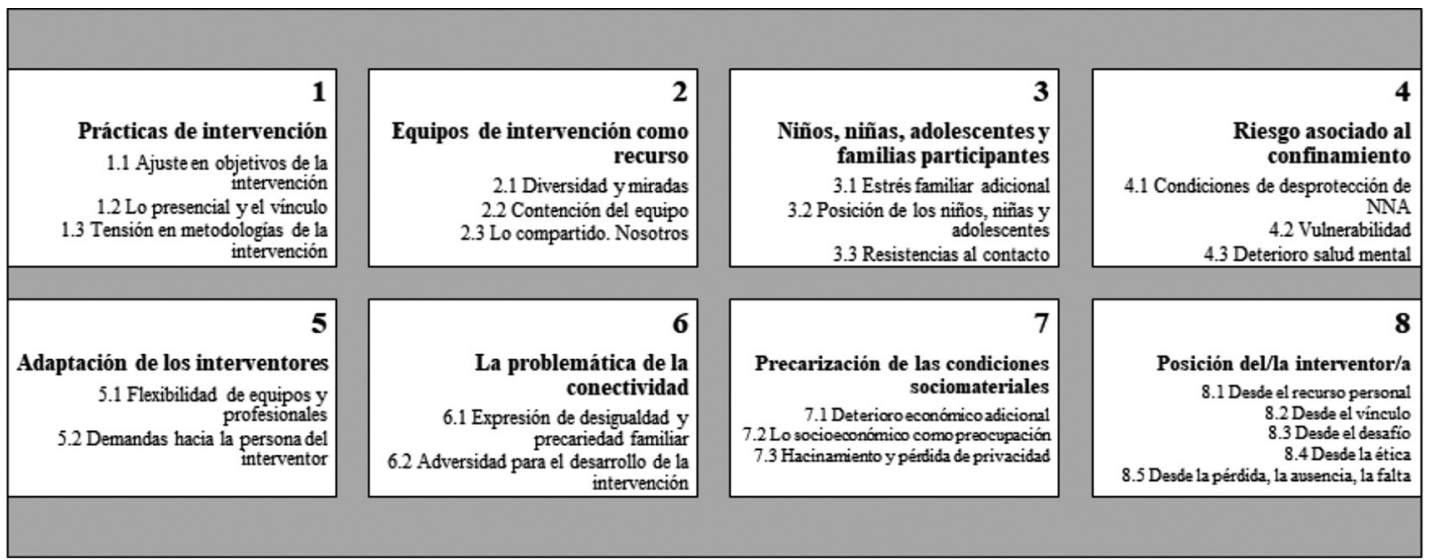


A continuación se realiza la descripción detallada de categorías y subcategorías identificadas.

\section{Categoría 1: Prácticas de intervención}

En esta categoría los participantes destacan y describen movimientos que han realizado en sus actividades y modos interventivos a partir del contexto de crisis sociosanitaria COVID-19, los que se organizan en torno a tres subcategorías:

Subcategoría 1.1: Ajuste en objetivos de la intervención. Alude a la revaloración de los objetivos interventivos que tenían en los procesos de los niños, niñas y adolescentes y que han cuestionado y redefinido con distintas orientaciones.

\footnotetext{
"Hay que saber adaptar los objetivos de atención considerando ahora a la contingencia salud" (participante 33).

"Las mismas sesiones de videollamadas, que ahora promueven objetivos de intervención más que seguimiento como al principio" (participante 12).
}

Subcategoría 1.2: Lo presencial y el vínculo. Agrupa los significados que establecen la relación entre el carácter presencial de la intervención, que apunta a los momentos previos a las crisis, a las familias en que hubo intervenciones presenciales, o a la falta de ellas, relacionándolas principalmente con el vínculo interventivo como una experiencia en tensión en la actualidad.

"Uno de los efectos que veo es la fragilización del proceso interventivo presencial" (Participante 27).

"Hoy mantener el vínculo a distancia es muy difícil" (Participante 26).

"La ausencia de intervención directa puede entorpecer el proceso reparatorio del niño" (Participante 29).

Subcategoría 1.3: Tensión en metodologías de intervención. Se relaciona con la tensión con la que hoy en la crisis identifican los interventores el uso, adecuación, limitaciones y posibilidades de las metodologías interventivas.

"Creación de recursos audiovisuales para la intervención hoy son necesidad" (Participante 42).

"Hay dificultad en desplegar estrategias, materiales y recursos de intervención" (Participante 29).
"En teleintervención que los niños no nos visualicen como invasores de su intimidad" (Participante 5).

\section{Categoría 2: Equipos de intervención como recurso}

En esta categoría se agrupan los significados que emergen en los participantes asociados al equipo como colectivo, el que emerge positivamente en cuanto a su valoración como recurso, agrupándose en torno a tres subcategorías:

Subcategoría 2.1: Diversidad y miradas. Incluye los significados que distinguen las posibilidades de contar con opiniones y perspectivas diversas al enfrentar el actual escenario de intervención, lo que se valora positivamente.

\begin{abstract}
"El trabajo en equipo favorece las intervenciones de los niños y niñas" (Participante 30).

"Es un recurso entender que todos somos responsables de la intervención y todos podemos aportar" (Participante 7).
\end{abstract}

Subcategoría 2.2: Contención del equipo. En esta subcategoría se incorporan los significados que destacan las valoraciones positivas de distintos modos de apoyo entre los distintos miembros del equipo, como una forma de ser y estar en equipo.

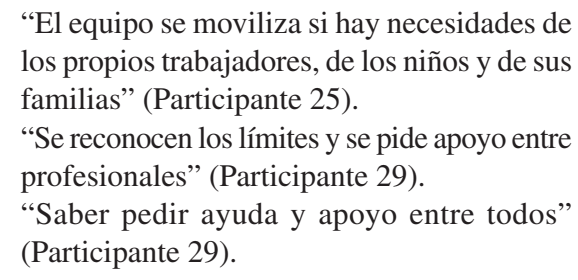

Subcategoría 2.3: Lo compartido. Nosotros. Esta subcategoría alude a los significados que enfatizan la posición de quien habla desde un lugar compartido (no individual) que incluye distintas expresiones para distinguirla, todas positivas, que marcan este colectivo emergente.

\footnotetext{
"La voluntad, el compromiso y el cariño hacia el trabajo que tenemos todos" (Participante 39). "La fuerza para sacar adelante los obstáculos entre todos" (Participante 33).

"Vernos y estar atentos entre todos a lo que vaya pasando, como solidaridad" (Participante 29).
} 


\section{Categoría 3: Impacto en niños, niñas, adolescentes y familias participantes}

En esta categoría se describen las expresiones que los participantes sostienen en torno a las vivencias de los niños, niñas y adolescentes, así como de sus familias frente a la actual crisis sociosanitaria. Los significados que emergen se agrupan en tres subcategorías.

Subcategoría 3.1: Estrés familiar adicional. En esta subcategoría se destaca la adición de nuevas fuentes de estrés que sobrecargan las condiciones desmejoradas que ya experimentaban los adultos a cargo de los cuidados de los niños, niñas y adolescentes, dificultando las posibilidades de responder de manera adecuada a las demandas que conlleva su rol parental.

"Se aprecian situaciones de estrés y angustia en los adultos responsables lo que generaría estados de sobrepaso en el proceder parental en torno a las prácticas de cuidado y crianza, elementos los cuales tienden a ser precipitados por diferentes razones, siendo muchas de éstas poco controlables o manejables por estos, como por ejemplo: desempleo definitivo o temporal, dificultades en el ámbito económico y/o socio-habitacional, problemas conductuales en sus hijos, antecedentes de salud mental, etc)" (Participante 22).

"La presencia de altos niveles de estrés en adultos asociado al período de confinamiento, estrés parental, dificultad para regularse emocionalmente y regular a los NNA del hogar, escasas redes de apoyo familiar sobre el cuidado y supervisión de los NNA, procesos clínicos suspendidos al igual que sus tratamientos farmacológicos, cesantía, merma en los ingresos económicos, por ende, dificultades para cubrir las necesidades básicas del grupo familiar" (Participante 21).

Subcategoría 3.2: Posición de los niños, niñas y adolescentes. Esta subcategoría alude a las afirmaciones de los participantes en torno a la particular vivencia de los niños, niñas y adolescentes en torno al contexto de crisis actual, que se expresa en diferentes dominios de su desarrollo, así como en los modos de implicarse en la atención brindada por los profesionales.

"En algunos adolescentes, se advierte aumento de sintomatología (principalmente cuadros ansiosos, depresivos, conductas autolesivas, desregulación emocional y conductual), asociado al estrés por confinamiento, pérdida del contacto directo con amistades y por carencia de espacios recreativos y de socialización" (Participante 21).

"En relación a los adolescentes, suelen preferir el contacto por WhatsApp, hay semanas que no atienden el teléfono y otras sí, pero me he dado cuenta que prefieren escribir, el obstáculo de eso, es que escriben cuando quieren, es decir, yo puedo escribir a las 12 del día y ellos me responden a las 15 horas yo respondo y ellos me responden al día siguiente, como aspecto positivo, suelen profundizar y ser más reflexivos en sus respuestas" (Participante 11).

Subcategoría 3.3: Resistencias al contacto. Junto con las anteriores, los participantes destacan la respuesta de los adultos, niños, niñas $\mathrm{y}$ adolescentes ante la continuidad de los procesos de intervención en el contexto COVID-19, que en algunas oportunidades genera oposición o rechazo a ciertas modalidades de acompañamiento.

"Reticencias en algunos casos para continuar con el proceso o generar instancias de comunicación que no sean telefónicas" (Participante 17).

"Reticencias en algunos casos para continuar con el proceso" (Participante 16).

\section{Categoría 4: Riesgo asociado al confinamiento}

En esta categoría se agrupan las expresiones de los participantes en torno a factores de riesgo adicionales, que emergen en las actuales condiciones de confinamiento y que se encuentran en algunas de las familias con las que trabajan. Los significados que aparecen se agrupan en tres subcategorías.

Subcategoría 4.1: Condiciones de desprotección de NNA. Esta subcategoría agrupa los significados que emergen en los participantes en torno al incremento de la condiciones de desprotección en que se encuentran algunos niños, niñas $\mathrm{y}$ adolescentes, asociados al aislamiento social que involucra el confinamiento, impidiendo el resguardo de su seguridad e invisibilidad de potenciales nuevas graves vulneraciones a las que estén expuestos.

"Factor de riesgo para nuestros usuarios, más aún en los casos en los que el ingreso del NNA 
se vincula con violencia intrafamiliar o bien viven con su presunto agresor" (Participante 12) "casos que jóvenes que han tenido que recurrir a prácticas de explotación sexual debido a la crisis" (Participante 18).

Subcategoría 4.2: Vulnerabilidad. En esta subcategoría los participantes destacan la presencia de condiciones contextuales impuestas por la crisis sociosanitaria, que fragilizan los recursos disponibles en las familias, e incrementa la vulnerabilidad psicosocial que poseían previo a esta contingencia.

\begin{abstract}
"Podemos identificar a su vez, las consecuencias adversas que ha traído consigo la propagación del virus de la COVID-19, como lo son, no contar con todas las instancias intersectoriales que también mantenían una visualización constante de los escenarios vulneradores de los NNA, esto debido a que, en su mayoría, las instituciones garantes de derecho nos encontramos en trabajo online" (Participante 4).

"A mi parecer los principales obstáculos dicen relación con que las familias que atendemos en su mayoría sino la totalidad es que tienen un alto grado de vulnerabilidad psicosocial y por tanto se han visto expuestos a mayor necesidad socioeconómica dado el contexto a nivel país que ha implicado que muchas familias no puedan trabajar o hayan sido cesados de sus puestos de trabajo y esto afecta a cada uno de los NNA" (Participante 32).
\end{abstract}

\section{Subcategoría 4.3: Deterioro salud mental.}

Esta subcategoría alude a las expresiones de los participantes en torno al impacto que el contexto COVID-19 ha generado en la integridad psicológica de los niños, niñas y adolescentes, así como sus adultos a cargo. Del mismo modo, se releva la limitación en la respuesta sanitaria existente para abordar la emergencia de esta demanda y la continuidad en el tratamiento de casos preexistentes y que agudizaron su sintomatología.

"En este contexto, se aprecia, que la salud mental se ha visto afectada, de tal manera, que han aumentado significativamente los niveles de angustia, estados depresivos, labilidad emocional, dificultades para dormir, alteraciones alimenticias. Es importante mencionar que, muchos casos que se atienden ya cuentan con antecedentes en salud mental, los cuales, se agudizan en el contexto de pandemia" (Participante 8).

\begin{abstract}
"Muchos usuarios cuentan con enfermedades de salud mental de base, algunas sin tratamiento, y dada la pandemia muchos de estas enfermedades se han acrecentado considerablemente, aumentando la inestabilidad emocional de los usuarios" (Participante 19).
\end{abstract}

\section{Categoría 5: Adaptación del interventor/a}

En esta quinta categoría se recogen y agrupan aquellas expresiones de los/as participantes en donde se enfatizan distintos niveles de ajuste y cambio que la situación de pandemia COVID-19 les implica y demanda, tanto en relación con el funcionamiento personal como con sus prácticas de intervención. Se identifican 3 subcategorías centrales:

\section{Subcategoría 5.1: Flexibilidad de equipos} y profesionales. En el escenario de pandemia, los participantes relevan la flexibilidad como una condición deseable y necesaria para su adecuado afrontamiento. El tópico se encuentra asociado a una evaluación más bien positiva y deseable, reconociéndose como característica, recurso y cualidad, tanto personal como del equipo de trabajo.

"Flexibilidad, dentro de las acciones que
realizo he tenido que usar bastante este recurso
últimamente. Las condiciones van cambiando
y la incertidumbre con respecto a la situación a
nivel mundial hace que uno vaya planificando
el día a día y también vaya entendiendo que
son situaciones que requiere un esfuerzo y
comprensión extra“ (Participante 1).
"Considero que principal recurso es la
flexibilidad que me ha permitido adaptarme a
las necesidades de la familia" (Participante 9).

Subcategoría 5.2: Demandas hacia la persona del interventor. En esta subcategoría los/as participantes describen y ejemplifican situaciones que les tensionan y desafían, en cuanto expresan requerimientos de articulación y adecuación entre necesidades asociadas a las nuevas modalidades de intervención y sus condiciones personales de vida.

\footnotetext{
"Por el lado de lxs trabajadores, también creo que ha sido un desafío, en primer lugar, traer a nuestro espacio de intimidad el trabajo y todo lo que eso puede implicar a nivel emocional. Por otro lado, transar en los límites de nuestra privacidad frente al uso de nuestros teléfonos para el contacto con las familias y la exposición de nuestra intimidad en las mismas. Sumado
} 
a congeniar el teletrabajo con las rutinas del hogar" (Participante 3).

"Reorganización de los tiempos de trabajo y tener presente los tiempos personales para la realización de la terapia on line sobre todo cuando se está desde casa y los tiempos de las familias que atendemos ya que en algunos casos sus rutinas se han visto alteradas" (Participante 7).

\section{Categoría 6: La problemática de la conectividad}

En el marco de la implementación de una modalidad de trabajo mayoritariamente remota, la temática de la conectividad adquiere una posición bastante central en el relato de los participantes. Un aspecto a destacar es la connotación más bien negativa que esta presenta, por cuanto los significados compartidos se relacionan más bien con la idea de una condición que dificulta el accionar de los interventores y de los NNA y familias con los que trabajan. Las subcategorías identificadas en este caso corresponden a 2 :

Subcategoría 6.1: Expresión de desigualdad y precariedad familiar. Esta subcategoría recoge expresiones en torno a la idea de que la conectividad es un ámbito en donde se expresan y materializan, condiciones de precariedad material y económica propias de las condiciones de vida de las familias con las que se trabaja, y que dan cuenta de una situación de desigualdad en el acceso a este tipo de servicios y recursos.

"El que no todos los NNA y sus familias cuentan con acceso a redes sociales, al menos de forma permanente" (Participante 5).

"La desigualdad a la que se ven expuestas nuestras familias más vulnerables en el acceso de las familias a tecnología (internet, computador y telefonía) así como un analfabetismo virtual de los cuidadores de la tercera edad a cargo de niños pequeños que no les permiten tener mejores alternativas de atención en este contexto de pandemia" (Participante 7).

Subcategoría 6.2: Adversidad para el desarrollo de la intervención. La presente subcategoría agrupa expresiones relacionadas con la idea de que la conectividad y sus condiciones de operación, constituyen un contexto que se hace compleja y dificulta el trabajo con NNA y sus familias, asociándose mayoritariamente con la noción de obstáculo, y en menor medida como desafío.

"Considero que uno de los mayores desafíos de dicha atención podría ser el tiempo, espacio y conectividad con la que cuentan las familias, puesto que esto puede ser un factor obstaculizador al momento de concentrarse y conectarse en el vínculo terapeuta-paciente" (Participante 4).

"La intervención de manera remota propiamente tal, ya que en el contexto en el cual residen los niños y sus familias en ocasiones no es el más adecuado para la intervención” (Participante 6).

\section{Categoría 7: Precarización de las condiciones sociomateriales}

La presente categoría aglutina ideas en torno a las condiciones de vida de NNA y sus familias en el contexto de la emergencia sanitaria por COVID-19, destacando principalmente dimensiones económicas, materiales y sociales. Las asociaciones manifestadas expresan una evaluación mayoritariamente negativa, predominando las ideas de precariedad, vulnerabilidad y preocupación. Los contenidos agrupados se presentan en 3 subcategorías:

\section{Subcategoría 7.1: Deterioro económico} adicional. El significado predominante en la presente subcategoría, da cuenta de que el actual contexto constituye una condición que incrementa las condiciones de precariedad y vulnerabilidad sociomaterial en la que se encuentran las familias con las que se trabaja.

\begin{abstract}
"Creo que el principal obstáculo ha sido la situación socio-económica de los grupos familiares, visualizando que varios de los adultos a cargo han perdido su empleo o no han podido realizar actividades laborales de manera informal, que presentan regularmente. He identificado que esta situación ha sido el factor que ha generado mayor tensión y estrés a nivel familiar" (Participante 3).

"Necesidades básicas no cubiertas, lo cual aumenta el estrés familiar" (Participante 10).
\end{abstract}

Subcategoría 7.2: Lo socioeconómico como preocupación. El foco temático de esta subcategoría se centra en la apreciación que interventores realizan acerca de la irrupción y presencia de los temas socioeconómicos en la situación de NNA y familia. Se relevan tópicos como deterioro en la 
salud mental, preocupaciones constantes por temas económicos, dificultades para satisfacer necesidades básicas, entre otras.

"Trabajar bajo un contexto ya adverso, donde no existe una normalidad, por lo cual las familias y los niños tendrán un grado extra de ansiedad, temor e incertidumbre, agregándole las situaciones con las cuales viven día a día" (Participante 6).

"Mientras que en el caso de los niños y niñas, alta preocupación por enfermar o que su familia se enferme, temor a no ver a su familia, frustraciones asociadas al cumplimiento de labores escolares, aburrimiento propio asociado al confinamiento"(Participante 16).

Subcategoría 7.3: Hacinamiento y pérdida de privacidad. Esta subcategoría aglutina expresiones de los participantes relacionadas con las condiciones en la que transcurre la cotidianeidad de la vida familiar y las características de los espacios en los que se desarrolla el contacto con los NNA por parte de los interventores, debido a las condiciones de confinamiento y trabajo remoto. De este modo, por un lado se resalta el hecho de que no existen condiciones espaciales adecuadas para que la familia pueda convivir adecuadamente, y por otro, que estas impactan negativamente en las posibilidades de que NNA cuenten con espacios protegidos y de intimidad, para abordar sus problemáticas con los/ as interventores.

\begin{abstract}
"Sobre todo en aquellos casos con ingresos recientes, se aprecian pocos espacios de contacto con los niños y niñas en espacios privados, adultos hipervigilantes e inoculación de relatos" (Participante 2).

"Otro obstáculo, hace referencia a que, al momento de mantener contacto con el NNA, suele encontrarse con más niños o adultos, sin disponer de un espacio más tranquilo, lo que dificulta poder intervenir desde objetivos más elaborados, dando prioridad a vinculación y contención del niño" (Participante 11).
\end{abstract}

\section{Categoría 8: Posición del/la interventor/a}

En esta categoría se hace referencia a los "lugares" o disposiciones a partir de los/las cuales son descritas las acciones realizadas por el/la interventor/a, y que aluden al enfrentamiento a las consecuencias de la crisis sociosanitaria COVID19, distinguiéndose para ello cinco subcategorías:
Subcategoría 8.1: Desde el recurso personal. En este sentido se identifica la posición del/la interventor/a como un lugar interno, propio, reconocido en sí mismo/a y vinculado a sus habilidades, competencias, dentro de estas se identifican, entre otras, el conocimiento de sí, la capacidad creativa, empatía, tolerancia a la frustración, capacidad de adaptación y flexibilidad como recursos significativos:

\begin{abstract}
"Paciencia, comprensión y empatía respecto de poder comprender ciertas situaciones que se generan en la intervención con las familias y/o también en la realización del teletrabajo" (Participante 39).

"Los recursos personales de los profesionales en cuanto a flexibilidad, adaptación e innovación para la generación de intervenciones eficaces" (Participante 29).
\end{abstract}

Subcategoría 8.2: Desde el vínculo. La posición del/la interventor/a, es identificada desde un lugar relacional, que sostiene o no la intervención a partir del tipo de contacto establecido con NNA o familia, apareciendo el vínculo o la vinculación como un elemento aglutinador de la experiencia de pandemia o telecomunicación presente:

\footnotetext{
"Vinculación terapéutica con las familias y niños(as), ha favorecido que estos muestren apertura a establecer distintas formas de comunicación, en este caso telecomunicación" (Participante 10).

“...falta de vinculación previa con los niños y niñas siendo solo más factible con adolescentes" (Participante 2).
}

Subcategoría 8.3: Desde el desafío. El accionar del/la interventor/a aparece orientado tanto desde un lugar interno de demandas o necesidades, como desde un lugar externo, asociado a los requerimientos generados por las circunstancias adversas:

"Pienso también que un desafío importante en esta nueva realidad es poder realizar las labores propias de nuestro rol en base a la autodisciplina" (Participante 37).

"En cuanto a desafíos a considerar... Trabajar bajo un contexto ya adverso, donde no existe una normalidad, por lo cual las familias y los niños tendrán un grado extra de ansiedad, temor e incertidumbre, agregándole las situaciones con las cuales viven día a día" (Participante 6). 
Subcategoría 8.4: Desde la ética. El lugar del/la interventor/a aparece asociado a la adherencia a normas o principios humanos, profesionales, laborales, que parecieran estar dirigiendo, organizando o mentalizando su actuar; denotados en valores personales o profesionales trascendentes, incluyendo dentro de estos el respeto, el amor, la responsabilidad, la participación, el profesionalismo, "lo humano o humanizante" en su ejercicio laboral:

"Realizar trabajo humanizante... profesional promotor de trabajo humanizante" (Participante 17).

"Amor y oportunidad por y para, dedicarme a mi trabajo" (Participante 34).

Subcategoría 8.5: Desde la pérdida, la ausencia, la falta. El lugar del/la interventor/a alude a la valoración de un conjunto de circunstancias externas adversas que denotan estados de carencia en distintos grados, los que atraviesan la experiencia interventiva abarcando multiplicidad de ámbitos, tales como recursos básicos, condiciones materiales, personales, tecnológicas, metodológicas, de seguridad, que aparecen ya sea no presentes, insuficientes, cambiantes, insatisfechas, peligrosas, riesgosas, limitadas, no disponibles, entre otras, que deben ser enfrentadas y vivenciadas por los/las profesionales y los/las participantes en la cotidianeidad cambiante de la intervención.

\footnotetext{
"Por otro lado, poder adaptarnos a esta forma de intervención, no contando en ocasiones con estrategias y/o material para realizar actividades vía online" (Participante 3 ).

“...el hacinamiento no lo permite, por lo que la atención pierde privacidad, no configurándose completamente como un espacio seguro y personal, por ejemplo, para realizar contención emocional" (Participante 16).

“...algunos conviviendo con los agresores, ya que no se están cumpliendo las medidas cautelares" (Participante 19).
}

\section{DISCUSIÓN}

Una posible lectura interpretativa de los hallazgos obtenidos, permite identificar la constitución de un campo semántico que tiene como trasfondo central la idea de crisis (Martínez, 2020), asociado a los efectos y repercusiones que la pandemia por COVID-19 ha presentado para las familias e interventores respecto del desarrollo de su cotidianeidad, y especialmente en lo que refiere al desarrollo de los procesos de intervención. De este modo, destacan como ejes articuladores del campo, un eje relacionado con la identificación de participantes, destacando las posiciones de "familia-NNA" y otra de "interventor", como posición/sujeto que son reconocidos como actores centrales de la situación abordada. Destaca el hecho de que los participantes están permanentemente vinculados y relacionados al proceso de intervención desplegado, destacando de este modo una aproximación más relacional y procedimental, que esencialista. El segundo eje identificado se relaciona con la noción de vulnerabilidad-protección, en la que se destacan posiciones de riesgo, tensión y conflicto, las que contrastan por oposición, con un polo que releva las ideas de recursos, adaptabilidad y ajuste. De este modo, es posible representar el campo semántico emergente en el presente estudio como una matriz constituida por cuadrantes, que relacionan a participantes con factores de vulnerabilidad-protección. El aspecto anteriormente señalado resulta ampliamente compatible con el tratamiento que desde la psicología se han establecido como directrices para el abordaje de situaciones de crisis, en los que tienden a relevarse los eventos desencadenantes y las estrategias de enfrentamiento (Martínez, 2020).

Como una manera de representar el campo de significados obtenidos, se presenta un esquema a modo de síntesis integradora, por medio de la Figura 2.

En el relato de los entrevistados, las familias y NNA son principalmente asociados a significados y posiciones relacionadas con el polo de la vulnerabilidad, y por tanto en condición de riesgo; de este modo, tres de las ocho categorías identificadas ("familias, NNA participantes", "riesgo asociado al confinamiento" y "precarización de las condiciones sociomateriales") destacan la crisis sanitaria como un contexto que ha impactado negativamente en la situación de los beneficiarios de la intervención de los proyectos, en los que se relevan aspectos tales como intensificación de precariedad económica, desprotección ante situaciones de violencia en espacios íntimos, y amplificación de sintomatología previa. En este sentido, destaca el hecho de que no se identifican en los relatos obtenidos, categorías que relacionan a las familias y NNA con recursos o capacidades de afrontamiento. Otras dos categorías se encuentran fuertemente asociadas a contenidos de tensión, conflicto y desafío, pero se focalizan 
Figura 2

Esquema gráfico integrador de contenidos. Elaboración propia

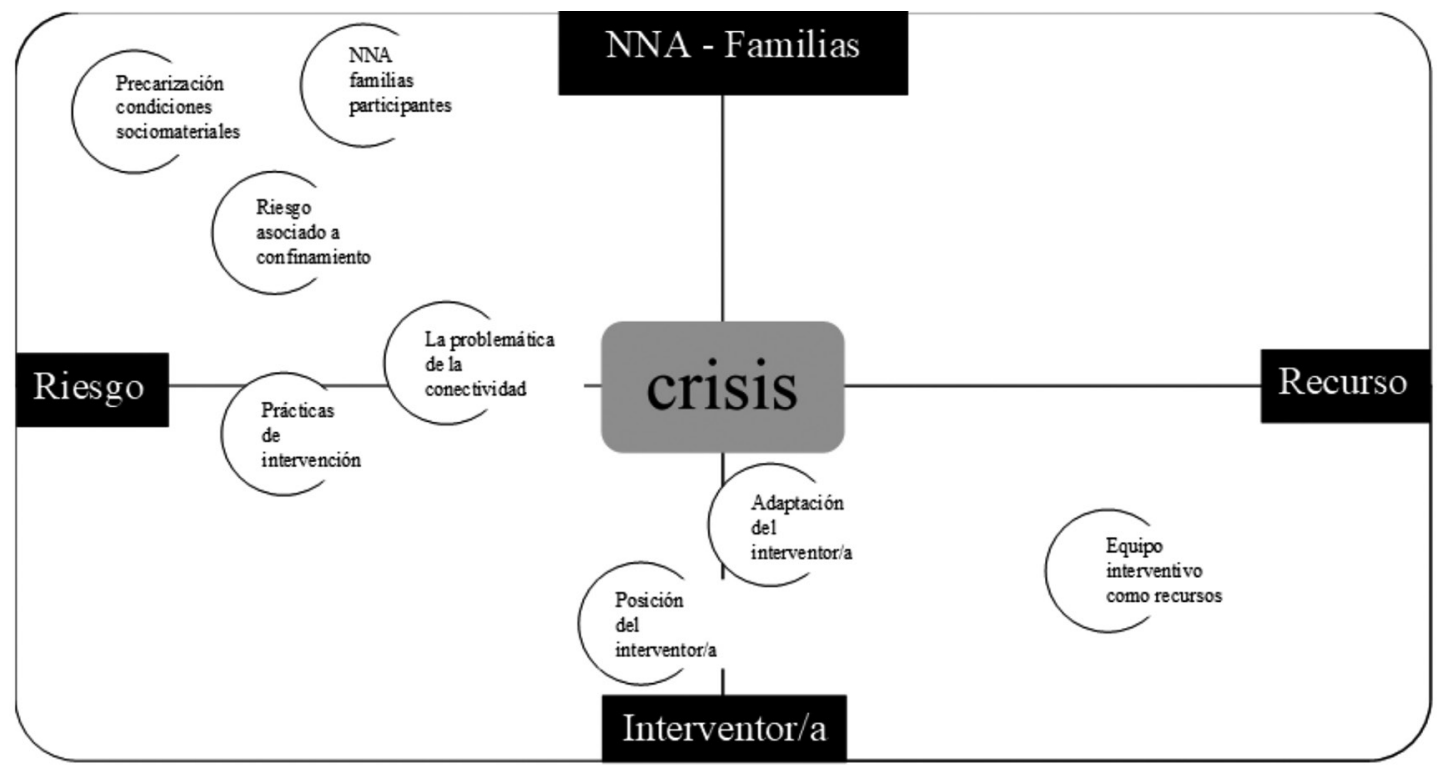

más bien en el proceso de intervención, que en los participantes propiamente tales; de este modo, "prácticas de intervención" y "problemática de la conectividad" son categorías que dan cuenta de cómo el actual escenario de pandemia se ha constituido en un contexto en el que el confinamiento aparece claramente como un factor de riesgo para la salud mental, se hacen complejas las prácticas y modalidades de intervención, y destacan los desafíos necesarios de asumir para asegurar su continuidad, en modalidades de vinculación mayoritariamente virtuales o remotas. Precisamente, la exploración de las modalidades de intervención psicosocial y psicoterapéutica en contexto de crisis y mediadas por tecnología, constituyen un área de interés para posibles estudios futuros (Soto-Pérez; Franco, Monardes y Jiménez, 2010; Vera-García, Castro y Caicedo, 2019).

De este modo, la mayoría de las categorías identificadas (cinco de ocho) reflejan valoraciones negativas para describir el actual estado de situación de las cosas, y resultan compatibles con hallazgos previos acerca del impacto y representación de la pandemia COVID-19 (Danese et al., 2019; Brooks et al., 2020; Dalton, Rapa y Stein, 2020; APA, 2020; United Nations, 2020). En este sentido, la interacción entre las dimensiones objetivas y subjetivas de la situación de crisis sanitaria, permite sostener que dentro de su multiplicidad de significados posibles, los/as interventores/as han resaltado las dimensiones de "dificultad, riesgo y peligro", por sobre las de oportunidad (González de Rivera y Revuelta, 2001; Bustos, 2005). Si bien el predominio de este tipo de perspectivas puede contribuir al desarrollo de un abordaje empático y comprensivo de la situación que experimentan NNA y sus familias, el predominio de la "perspectiva de riesgo" puede tener implicancias respecto de la eficacia de la intervención terapéutica, al limitar la capacidad del/la interventor/a para reconocer, reflejar y movilizar los recursos de los/as afectadas para el afrontamiento de la situación de crisis. Lo anterior, resulta especialmente relevante para la planificación de las etapas de la intervención, así como la adecuada distinción entre "intervención en crisis" y "psicoterapia en crisis" (Martínez, 2020).

Respecto de la situación de los interventores, los relatos registrados tienen a dar cuenta de sujetos que transitan entre condiciones de vulnerabilidad a condiciones de mayor ajuste, evidenciando capacidades y recursos de afrontamiento. Dos de las categorías identificadas ("adaptación de los interventores" y "posición de los interventores") describen cómo estos se encuentran tensionados y 
desafiados por las actuales condiciones de funcionamiento, pero al mismo tiempo destacan y resaltan recursos individuales y colectivos que les permiten enfrentar los profundos cambios acontecidos en los modos de desarrollar las prácticas de intervención y las formas de vinculación con las familias con las que se trabaja.

Estos aspectos son aún más resaltados por medio de la categoría "equipo de intervención como recurso", única categoría asociada en su integridad a contenidos positivos. En esta se aprecia como mayoritariamente los espacios de interacción con los integrantes del equipo, la relación con jefaturas directas y supervisores/as técnicos, son apreciados como espacios de contención y apoyo social, en que la diversidad de perspectivas y experiencias se valoran como recursos que enriquecen las posibles respuestas a entregar y expresan un sentido de identidad y pertenencia al colectivo inmediato, que es visualizado claramente como un recurso.

Si bien se destacan y resaltan aspectos individuales de afrontamiento y respuesta a la crisis en los interventores (flexibilidad, creatividad, compromiso e innovación), resulta importante destacar este relevamiento de las dimensiones colectivas y compartidas que sugiere la centralidad de la posición que el equipo alcanza como significación, siendo posible, en principio, relacionarla con dimensiones más colectivas de procesos tales como las sostenidas por la resiliencia comunitaria u organizacional (Pacheco-Mangas, 2014; Menanteux, 2015; Cossio, Ortega y Padrón, 2017). Estos hallazgos resultan solo parcialmente consistentes con estudios anteriores (Peralta-Gómez, 2011: Pacheco-Mangas, 2014), ya que no se replica una subjetividad de los interventores (trabajadores) configurada como disponibles, individualistas, competitivos y autoexigentes en el desempeño del trabajo. En este sentido, la exploración de las variables institucionales y de cultura organizacional/equipo participantes en la relevancia alcanzada por las dimensiones de recursos y resiliencia, aparece como una línea de investigación aportativa a futuro.

\section{Implicaciones prácticas}

Chile es un país que enfrenta habitualmente otras crisis -naturales y recientemente sociopolíticas- que si bien son diferentes a la crisis sociosanitaria por COVID-19 igualmente presentan ciertas similitudes como la restricción de movimiento, el estado socioemocional de alerta, el aislamiento social, etc. De esta manera, los hallazgos de este estudio pudiesen ser de utilidad en futuras crisis que tengan estas características. Esto ayudará a los equipos de atención a NNA vulnerados en sus derechos, a las instituciones que los administran y a los organismos estatales que los financian de forma de crear protocolos de atención en contextos de crisis que -por un lado- aseguren la atención de los NNA, y -por el otro- consideren los peligros y estresores adicionales propios de la crisis. Es sabido que la crisis del COVID-19 obligó a estas instituciones y organismos a tomar medidas sobre la marcha, ahora después de avanzada la crisis y en coherencia con los resultados obtenidos en este estudio se debería tener mayores elementos para enfrentar futuras crisis. En particular, resulta relevante fortalecer los mecanismos internos de coordinación de los equipos, protocolizar o sistematizar las estrategias de intervención que resultaron efectivas, además de establecer planes de protección y autocuidado para los profesionales, de forma de estar más preparados para sucesos futuros de las mismas características. Además, es claramente pertinente que los centros tengan planes de intervención en crisis para enfrentar situaciones similares que contemplen los nuevos riesgos de vulneración de derechos de los NNA y mecanismos para prevenir y detectarlos. Es importante también asegurar el trabajo en red que permita a las distintas instituciones que intervienen una misma familia o comunidad coordinarse para administrar eficientemente los recursos, considerando las dificultades de conectividad de las familias más vulnerables y la precarización de las condiciones materiales en las que viven producto de la crisis.

\section{CONCLUSIÓN}

A base de lo señalado, este estudio permite concluir que -aun en condiciones tan extremas como la vivida durante la crisis por COVID-19- los avances de la tecnología, la preparación, motivación y trabajo en equipo de los profesionales encargados de velar por los derechos de los NNA permiten realizar intervenciones en favor de esta población. Sin embargo, el trabajar con menos recursos, así como el mayor aislamiento y estrés de la familia, hacen que se requiera mayor atención ante eventuales nuevas vulneraciones o revictimizaciones que afecten a NNA en contexto de pandemia o crisis similares. 
Este estudio da cuenta de los desafíos de la intervención con NNA en el contexto proteccional chileno y deja abiertos varios desafíos para enfrentar crisis como la del COVID-19 sin que ello afecte a niños, niñas y adolescentes en condiciones de vulnerabilidad. Si bien es cierto este estudio releva la importancia de considerar los "riesgos" de la crisis, también deja ver algunas "oportunidades", especialmente a nivel vincular, relacional. No obstante, este estudio -debido a que fue realizado en una etapa inicial de la pandemia-no profundizó en las modalidades, metodologías o experiencia de intervención exitosas. Ahora -a más de un año de iniciada la pandemia- y en el futuro, sería pertinente realizar estudios que sean capaces de describir, evaluar y sistematizar intervenciones que hayan resultado positivas para los NNA durante la pandemia, en la línea de la reciente publicación de la ONG Paicabi (Arredondo-Ossandón, 2020). Esta puede ser la oportunidad que genere esta crisis, la de modernizar o ampliar formas efectivas y eficientes de intervención que puedan servir en el futuro para promover los derechos de los NNA o ayudarlos en su proceso de reparación. Este ha sido un estudio preliminar, que debiera ser considerado como un paso inicial para la construcción de un sistema proteccional chileno preparado para enfrentar crisis como la del COVID-19 u otras. 


\section{REFERENCIAS}

Arredondo- Ossandón, V. (2020). Voces y colores Prácticas de intervención y reflexiones en el contexto de crisis sociosanitaria COVID-19. Intervención especializada en reparación de la violencia hacia los niños, niñas y jóvenes. https://drive.google. com/file/d/1wJmgPnwX9hotQY6Al-wGiNUFy-w9rx1B/view

Arbeláez, M., y Onrubia, J. (2014). Análisis bibliométrico y de contenido. Dos metodologías complementarias para el análisis de la revista colombiana Educación y Cultura. Revista de Investigaciones UCM, 14(23), 14 - 31

APA (2020). How COVID-19 may increase domestic violence and child abuse. https://www.apa.org/topics/covid-19/ domestic-violence-child-abuse

Bardin, L. (2002). El análisis de contenido. Madrid: Ediciones Akal

Bernal, C. (2006). Metodología de la investigación: Para administración, economía, humanidades y ciencias sociales. México: Ediciones Pearson

Blesa, A., Cabo de Guzmán, F. García, M., Gehring, R., Muñoz, P., Palacios, J., y Rodes, J. (2014). Guía de criterios básicos de calidad en la investigación cualitativa. Cuadernos Metodológicos ICUALI 1. UCAM, España.

Brooks, S. K., Webster, R. K., Smith, L. E., Woodland, L., Wessely, S., Greenberg, N., y Rubin, G. J. (2020). El impacto psicológico de la cuarentena y cómo reducirla: revisión rápida de las pruebas. Lancet, 395, 912-20. https://doi.org/10.1016/ S0140-6736(20)30460-8

Bustos, R. (2005). Desarrollo local y representación: El concepto de crisis. Diálogo Andino-Revista de Historia, Geografía y Cultura Andina. 25, agosto, pp 53-76. Universidad de Tarapacá, Chile.

Cossio, P.E., Ortega, A. y Padrón, A. (2017) La importancia de la resiliencia organizacional en el personal de salud. En Ortega Velázquez, A. (Coord.) Nuevos retos de las organizaciones encargadas de la salud en México. Cap. 3, pp. 31-45. México, SA de CV.

Crouch, J., y Behl, L. (2001). Relationships among parental beliefs in corporal punishment, reported stress, and physical child abuse potential. Child Abuse \& Neglect, 25(3), 413-419. https://doi.org/10.1016/S0145-2134(00)00256-8

Dalton, L., Rapa, E. y Stein, A. (2020). Protecting the psychological health of children through effective communication about COVID-19. The Lancet Child \& Adolescent Health. Publicación en línea. https://doi.org/10.1016/S2352-4642(20)30097-3.

Danese A, Smith P, Chitsabesan P., y Dubicka B. (2019) Child and adolescent mental health amidst emergencies and disasters. British Journal of Psychiatry. Publicación en línea: httpss://10.1192/bjp.2019.24

De la Cuesta, C. (2014) La calidad de la investigación cualitativa. De evaluarla a lograrla. Texto Contexto Enferm, Florianópolis, 2015 Jul-Set; 24(3): 883-90. https://doi. org/10.1590/0104-070720150001150015

Díaz-Bravo, Laura, Torruco-García, Uri, Martínez-Hernández, Mildred, \& Varela-Ruiz, Margarita. (2013). La entrevista, recurso flexible y dinámico. Investigación en educación médica, 2(7), 162-167. Recuperado de: http://www.scielo.org.mx/scielo. php?script=sci_arttext\&pid=S2007-50572013000300009\&1ng=es\&tlng=es.
Dorset Police UK (2020). Online grooming and child exploitation during COVID 19 lockdown. Recuperado de: https://www.dorset. police.uk/covid-19-coronavirus/cse-and-covid-19/

Earl, A. (2000). Fundamentos de la investigación social. México: Internacional Thomson Editores

Fernández, F. (2002). El análisis de contenido como ayuda metodológica para la investigación. Revista de Ciencias Sociales (Cr), 96: 35-53, (II)

Gracia, E. y Musitu, G., (2003). Social isolation from communities and child maltreatment: a cross-cultural comparison. Child Abuse \& Neglect, 27(2), 153-168. https://doi.org/10.1016/ S0145-2134(02)00538-0

González de Rivera y Revuelta, J.L. (2001). Psicoterapia de la crisis. Revista de la Asociación Española de Neuropsiquiatría. Año XXI, Julio/Sept, pp. 35-53.

González Rey, F. (2000). Investigación Cualitativa en Psicología. Rumbos y desafíos. México: International Thomson

Kuntz, S. (2008). Predicting patterns of interaction between parents and children based on parent reports of stress and potential for child maltreatment. Dissertation and Theses. Department of Applied Behavioral Science and the Faculty of the Graduate School of the University of Kansas

Martínez, C. (2020). Intervención y psicoterapia en crisis en tiempos del coronavirus (para psicólogos/as clínicos/as y psicoterapeutas).Centro de Estudios en Psicología Clínica y Psicoterapia de la Universidad Diego Portales. MIDAP. Santiago, Chile.

Monanteux, M.R. (2015) Resiliencia comunitaria y su vinculación al contexto latinoamericano actual. Cuadernos de Trabajo Social, 14, diciembre 2015, Universidad San Sebastián (Concepción, Chile), ISSN 0719-6520, pp.23-45

ONU (1989). Asamblea General, Convención sobre los Derechos del Niño, 20 Noviembre 1989. United Nations, Treaty Series, 1577, p. 3. https://www.refworld.org.es/docid/50ac92492.html

Pacheco-Mangas, J. (2014). Estrategias ante la adversidad: la resiliencia en el discurso de los profesionales de Servicios Sociales Comunitarios. En Pastor, E. (Edit.) (2014). El Trabajo Social ante el reto de la crisis y la educación superior (pp. 951958). Madrid: Universitas

Peralta-Gómez, M. (2012). Significados, cambios y contexto actual de trabajo.: Estudio interpretativo. Diversitas: perspectivas en psicología, 8(1), 165-182.

SENAME (2020). Resolución Exenta N 862 Aprueba Modificación Instructivo Intervención. Recuperado de: https://www.sename.cl/ web/wp-content/uploads/2020/03/Rex-862-Aprueba-modificacionInstructivo-Intervencion.pdf

Soto-Pérez, F., Franco, M., Monardes, C., y Jiménez, F. (2010). Internet y psicología clínica: Revisión de las ciberterapias. Revista de Psicopatología y Psicología Clínica Vol. 15, N. ${ }^{\circ}$ 1, pp. 19-37, 2010 ISSN 1136-5420/10. Recuperado de:

f i l e : / / / C : / U s e r s / c r a j c / D ow n loa d s / Internetypsicologaclnicarevisindelasciberterapiasversrevista.pdf

Tucker, M. y Rodríguez, C. (2014). Family Dysfunction and Social Isolation as Moderators Between Stress and Child Physical Abuse Risk. Journal of Family Violence, 29(2), 175-186. DOI:10.1007/s10896-013-9567-0 
United Nations (UN) (2020). COVID-19 Global Humanitarian Response Plan. Recuperado de: https://www.unescap.org/news/ covid-19-global-humanitarian-response-plan

UNICEF (2020). How to keep your child safe online while stuck at home during the COVID-19 outbreak. Recuperado de: https://www.unicef.org/coronavirus/ keep-your-child-safe-online-at-home-covid-19
Vera-García, B. M., Castro, J., y Caicedo, L. (2019). La intervención del psicólogo a través de las redes sociales:: Una perspectiva desde la ética.Didasc@ lia: Didáctica y Educación, 10(2), 133-146. 